\title{
Why My Manuscript Was Rejected? What Next?
}

Saurabh RamBihariLal Shrivastava*, Prateek Saurabh Shrivastava and Jegadeesh Ramasamy

Shri Sathya Sai Medical College \& Research Institute, Kancheepuram, Tamilnadu, India

*Corresponding author: Saurabh RamBihariLal Shrivastava, Department of Community Medicine, Shri Sathya Sai Medical College \& Research Institute, Ammapettai village, Thiruporur - Guduvancherry Main Road, Sembakkam Post, Kancheepuram - 603108, Tamil Nadu, India, Tel: +919884227224; Email:drshrishri2008@gmail.com

Rec date:October20, 2014; Acc date: October 22, 2014; Pub date:October 26, 2014

Copyright: (c) 2014 Shrivastava SR, et al. This is an open-access article distributed under the terms of the Creative Commons Attribution License, which permits unrestricted use, distribution, and reproduction in any medium, provided the original author and source are credited.

\begin{abstract}
The rejection of manuscripts is quite a common phenomenon, and multiple reasons have been identified, which in combination provides enough evidence to the editorial board / reviewers' to reach to their decision. The rejection letters generally serve two purposes, namely it proves that you have actually written up your work; and at the same time can be a source of good constructive criticism so that manuscripts can be significantly improved prior to submission to another journal. The authors should realize that they have received free suggestions from a qualified reviewer so that quality of the manuscript can be improved before it achieves publishable value. In conclusion, performing research and getting it accepted and published in a quality journal is not a walk in the park. However, in the research arena, manuscript rejection is a fact and thus authors need to be positive, persevere with their ideas, and continue their writing.
\end{abstract}

\section{Keywords: Manuscript; Rejection; Journal}

\section{Introduction}

Worldwide, in an attempt to improve the health standards and augment the understanding of human biology, multiple research studies have been conducted in the field of health sciences [1]. However, in order to communicate the research findings to the different corners of the world, the study findings are submitted by the researchers in the form of a manuscript (viz. research articles, brief reports, review articles, commentaries, meta-analysis, expert opinions, medical education, letters to the editor, notes from the field, technical note, editorials, etc.), to a national or international journal for publication [1].

These submitted manuscripts are assessed by the Editor-In-Chief and other editorial members of the journal, and based upon its suitability with the scope of the journal or fulfillment of the journals' guidelines, a decision is made to either reject the manuscript outrightly or sent for peer-review $[2,3]$. Once the reviewer comments are received, only the outstanding articles / articles with minor flaws are allowed to go to next stage, while rest all are declined by giving appropriate reasons for rejection $[1,2]$. In-fact, it has been reported that manuscript rejection is quite common (even higher in journals with high impact factor), and almost $62 \%$ of published manuscripts have been rejected at least one time [4,5].

Multiple reasons like submitting the manuscript to a wrong journal; non-compliance with the journal instructions to prepare manuscript (viz. abstract not written as desired, citation of references in the text and at the end, etc.); dearth of originality (viz. employment of obsolete methods, results that are unoriginal, predictable, trivial, lacks generalizability, and have no clinical implications); poor rationale for the study; wrong study design; inadequate description of the methods; small or inappropriately chosen sample; inappropriate statistical analysis; sub-optimal reporting of the results; lack of interpretation; inadequate literature review; citation of very old references; poor writing - improper formatting and language, grammatical lapses, and typographic errors; failure to revise and resubmit following peer review; inadequate corrections of galley proofs; simultaneous submission to another journal; and definitive evidence of plagiarism; have been identified, which in combination provides enough evidence to the editorial board / reviewers' to reach to decision of rejecting the manuscript $[1,4,6-9]$. In addition, factors like space constraints; quality and experience of peer reviewers; number of issues published per year; decision-making policy of the journal; the journal entertains only theme-based articles for different issues; and receipt of more than one submission on the same topic, further aggravates the problem of rejection $[7,10,11]$.

It has been proposed that manuscripts are either rejected on an outright basis (if there is no new information in the manuscript or a major methodological error); or are correctable (if there is error in manuscript organization or linguistic errors) $[2,6]$. The rejection letters generally serve two purposes, namely it proves that you have actually written up your work; and at the same time can be a source of good constructive criticism so that manuscripts can be significantly improved prior to submission to another journal [2]. Although, rejection of a manuscript is disappointing, nevertheless, most of them gets published within a reasonable time interval [4]. The authors should realize that they have received free suggestions from a qualified reviewer so that quality of the manuscript can be improved before it achieves publishable value [3]. Furthermore, as already stated above there are many causes for rejection and hence it should not be taken personally $[3,9]$.

In order to prevent the manuscripts from being rejected, some tips have to be followed, namely choose the journal carefully (viz. based on its scope, visibility, \& importance of the work done); look for the most recent instructions to authors; follow the instructions thoroughly; subject the manuscript for grammatical \& linguistic check; give more attention to methodology \& discussion section; cite appropriate \& recent references in the desired manner; and ask a colleague to critically assess your paper $[1,3,8,9]$. In addition, while replying to 
Citation: Shrivastava SR, Shrivastava PS, Ramasamy J (2014) Why My Manuscript Was Rejected? What Next?. Primary Health Care 4: e113. doi:10.4172/2167-1079.1000e113

Page 2 of 2

referees' comments, ensure that the response is quick, comprehensive, courteous, and evidence-based to increase the chance of acceptance of the manuscript $[12,13]$.

In conclusion, performing research and getting it accepted and published in a quality journal is not a walk in the park. Thus, authors should sincerely attempt to improve the quality of their work. However, in the research arena, manuscript rejection is a fact and thus authors need to be positive, persevere with their ideas, and continue their writing.

\section{References}

1. Ali J (2010) Manuscript rejection: causes and remedies. J Young Pharm 2: 3-6.

2. Woolley KL, Barron JP (2009) Handling manuscript rejection: insights from evidence and experience. Chest 135: 573-577.

3. Peregrin T (2007) How to cope with manuscript rejection. J Am Diet Assoc 107: 190, 192-193.

4. Hall SA, Wilcox AJ (2007) The fate of epidemiologic manuscripts: a study of papers submitted to epidemiology. Epidemiology 18: 262-265.

5. Kurmis AP, Kurmis TP (2006) Exploring the relationship between impact factor and manuscript rejection rates in radiologic journals. AcadRadiol 13: 77-83.
6. Wyness $\mathrm{T}$, McGhee $\mathrm{CNj}$, Patel $\mathrm{DV}$ (2009) Manuscript rejection in ophthalmology and visual science journals: identifying and avoiding the common pitfalls. Clin Experiment Ophthalmol 37: 864-867.

7. Pierson DJ (2004) The top 10 reasons why manuscripts are not accepted for publication. Respir Care 49: 1246-1252.

8. Ehara S, Takahashi K (2007) Reasons for rejection of manuscripts submitted to AJR by international authors. AJR Am J Roentgenol 188: W113-116.

9. Turcotte C, Drolet P, Girard M (2004) Study design, originality and overall consistency influence acceptance or rejection of manuscripts submitted to the Journal. Can J Anaesth 51: 549-556.

10. Henly SJ, Dougherty MC (2009) Quality of manuscript reviews in nursing research. Nurs Outlook 57: 18-26.

11. Garmel GM (2010) Reviewing manuscripts for biomedical journals. Perm J 14: 32-40.

12. Williams HC (2004) How to reply to referees' comments when submitting manuscripts for publication. J Am Acad Dermatol 51: 79-83.

13. Kotsis SV, Chung KC (2014) Manuscript rejection: how to submit a revision and tips on being a good peer reviewer. Plast Reconstr Surg 133: 958-964. 